Relations industrielles

Industrial Relations

\title{
La sécurité syndicale et le syndicat
}

\section{Gérard Picard}

Volume 2, numéro 8, avril 1947

URI : https://id.erudit.org/iderudit/1023873ar

DOI : https://doi.org/10.7202/1023873ar

Aller au sommaire du numéro

Éditeur(s)

Département des relations industrielles de l'Université Laval

ISSN

0034-379X (imprimé)

1703-8138 (numérique)

Découvrir la revue

Citer cet article

Picard, G. (1947). La sécurité syndicale et le syndicat. Relations industrielles / Industrial Relations, 2(8), 2-3. https://doi.org/10.7202/1023873ar

Tous droits réservés @ Département des relations industrielles de l’Université Laval, 1947
Ce document est protégé par la loi sur le droit d'auteur. L’utilisation des services d'Érudit (y compris la reproduction) est assujettie à sa politique d'utilisation que vous pouvez consulter en ligne.

https://apropos.erudit.org/fr/usagers/politique-dutilisation/ 


\section{LA SÉCURITÉ SÝNDICALE ET LE SYNDICAT}

Les organisateurs de la présente session du Congrès des relations industrielles de Laval ont détaché de la question sociale, et plus particulièrement de la question ouvrière, un sujet de grande actualité: la sécurité syndicale. La période que nous traversons ne paraît pas des plus propices à une étude poussée des droits que peuvent avoir les syndicats de travailleurs, vu que la presse quotidienne et les périodiques ont tellement monté en épingles, en ces derniers temps, certains conflits industriels et quelques remous de cette mer humaine qu'est la classe ouvrière. L'on peut expliquer, en partie, la situation en relisant un texte publie en 19:35, dans le rapport de la Commission royale d'enquète sur les écarts de prix, (p. 138), et dont l'actualité reste prenante, malgré la poussière d'oubli dont on l'a recouvert. Voici:

«La reconnaissance plus complète des syndicats ouvriers et par les gouvernements et par les patrons aurait une plus grande portée que de simplement faciliter la négociation collective des contrats de salaires. Tant que le syndicalisme ne sera que toléré, et nous avons la preuve qu'il en est souvent ainsi, il continuera dans ses tactiques défensives. Dans la mesure que les syndicats ouvriers seront reconnus comme instruments nécessaires à l'organisation et au contrôle économique, dans cette mesure les forces et l'intelligence du mouvement pourront être totalement libérées pour coopérer efficacement à l'amélioration des conditions sociales. C'est la psychologie défensive imposée aux Syndicats par l'expérience et les circonstances qui développe ses méthodes et pratiques auxquelles on s'oppose parfois, mais qui sont souvent employées par leurs adversaires pour créer des malentendus et des préjugés. »

La sécurité syndicale, si on la considère comme un simple moyen de faire du recrutement forcé de membres en faveur d'un syndicat, ou coinme un moyen de conserver intacts, pour une période déterminée, les effectifs cotisants ou le total des cotisations d'un syndicat, prend un sens matérialiste et totalitaire, et il faudrait, dans ce cas, le confier au patron des causes désespérées. Cette manière de voir, bien que répandue, doit être écartée. Une analyse plus approfondie de la question justifiera, espérons-le, des conclusions moins simplistes et plus conformes aux buts poursuivis par l'organisation syndicale des travailleurs. Sécurité syndicale: garantie exprimée dans une convention collective ou assurée par une sc ntence arbitrale liant les parties, et permettant à un syndicat de travailleurs, pour une période déterminée, de remplir efficacement son rôle et d'assumer toutes ses responsabilités sans que son existence ne soit mise en danger par l'employeur ou par certains employés.

Les principales formes sous lesquelles se présente la sécurité syndicale ne sont pas exclusives au point de ne pouvoir se multiplier en empruntant les unes aux autres, mais on accepte généralement les distinctions suivantes: 1.-L'atelier fermé (closed shop); 2.-L'atelier syndical (union shop); a) parfait; b) imparfait; 3.-le maintien d'affiliation (maintenance of membership); 4.-la préférence syndicale (preferential shop); 5.-la formule Rand; 6.-la retenue syndicale (check-off).

Les contrats d'atelier fermé sont fréquents aux Etats-Unis et au Canada, et il y en a un bon nombre dans la Province de Québec, notamment dans l'industrie du bâtiment, dans l'industrie de l'imprimerie, dans l'industrie de la fourrure, dans l'industrie de la confection pour hommes et garçons, dans l'industrie de la confection pour dames, dans les services hospitaliers, etc.

La formule Rand n'est acceptable que dans les cas où il n'y a que des syndiqués membres d'un même syndicat, et des non-syndiqués à l'emploi d'un même enıployeur. Et les «scabs » peuvent se compter chanceux de s'en tirer à si bon compte. Mais Ia formule Rand ne saurait être admissible si on voulait l'appliquer contre un syndicat minoritaire légitime, car ce serait violer la liberté syndicale et faire payer une double cotisation à des salariés qui ont choisi leur syndicat. La seule exception acceptable serait l'application de la formule Rand contre un syndicat minoritaire dominé par l'employeur et susceptible de mettre en dancer les intérêts professionnels des travailleurs. Il s'agirait alors d'un cas «d'union de compagnie », d'une association de «scabs».

Il n'est pas nécessaire d'étudier en détail toutes les distinctions et formalités qui se rattachent à la retenue syndicale. C'est au'onrd'hui une formule de sécurité syndicale qui passe dans les moeurs. et où les objections du début ont été résolues soit par négociations directes soit par jurisprudence arbitrale. L'em- ployeur bien disposé et prêt à coopérer vraiment avec un syndicat de travailleurs, qui considère ses employés et leur syndicat comme des associés, sait très bien qu'il rend service au syndicat en appliquant gratuitement la retenue syndicale, et il accepte de le farre, sachant que le syndicat recevra ainsi régulièrement les cotisations de ses membres, qu'il sera en meilleure posture financière, que ses officiers seront dégagés du problème de la collection, et qu'ils pourront remplir plus efficacement les devoirs de leurs charges

Le maintien d'affiliation et les diverses formes d'atelier syndical sont en réalité des compromis par rapport à l'atelier termé. Certains employeurs acceptent le maintien d'affiliation ou l'atelier syndical imparfait, parfois parce qu'ils considèrent respecter ainsi, dans toute la mesure possible, la liberté syndicale, et parfois, pour mieux faire comprendre leur opposition définitive à l'atelier fermé

Du point de vue syndical, que penser de l'atelier fermé ? Voilà le sujet qui sera traité dans la dernière partie de cette conférence.

Il ne s'agit pas ici d'anlyser, par comparaison, la situation des professionnels et celle des travailleurs, ni d'essayer d'établir que certaines restrictions nécessaires à la liberté individuelle posées pour être admis à l'étude et à la pratique des professions libérales peuvent trouver leur application, mutatis mutandis, en faveur de l'admission à l'apprentissage et à la pratique d'un métier. Pourquoi provoquer d'inutiles énervements et compliquer à l'extrême une situation qui peut être autrement dénouée?

Deux cents employés sont au service d'un méme employeur. Chacun de ces deux cents employés peut, en aucun temps, et sans donner de raison, remettre à l'employeur l'avis légal de séparation, remplir les dernières conditions prévues, et quitter son emploi. L'employeur peut congédier, sans donner de raison, un employé, après avoir observé les preseriptions légales en cette matière. Rien n'empêche les deux cents empłoyés d'agir collectivement; et rien n'empêche l'employeur d'effectuer un congédiement massif. Il peut y avoir embarras sérieux de part ou d'autre, mais si les employés ne sont pas syndiqués, personne ne portera attention à leur départ volontaire ou à leur congédiement. Mais dès que la vie syndicale circule dans un groupement de travailleurs, on sera mal inspiré de croire que tous les problèmes se règleront en portant un trèfle à quatre feuilles à la boutonnière. Quand l'employeur n'a devant lui que des travailleurs - isolés, il est de beaucoup le plus fort, et la partie n'est pas égale. Lorsque les travailleurs sont syndiqués, ils deviennent plus libres et, avec le temps, les négociations se font sur un pied d'égalité entre les parties. C'est peut-être plus fatigant, mais l'on satisfait mieux aux exigences de la justice sociale.

Les deux cents employés dont il a été question, il y a quelques instants, reviennent devant nous. Ils décident, en nombre substantiel, de former un syndicat professionnel. Personne ne saurait leur contester ce droit. Il serait inconvenant de traiter ici de l'ABC du droit d'association. C'est un droit naturel consacré dans notre législation. L'association professionnelle, pour les travailleurs, est nécessaire, non pas in se, mais de nécessité de moyen. D'ailleurs, la question de principe n'est même pas soulevée lorsqu'il s'agit des autres classes de la société.

Le syndicat vise à protéger les métiers, à assurer un salaire raisonnable et des conditions de salaires convenables. Lorsqu'il jouit de la personnalité civile. il pose le geste de sa responsabilité devant les lois du pays. " Mais il n'entre pas dans le cadre de la présente conférence de traiter ce sujet. Devant la législation actuelle, malheureusement, les syndicats sont placés sur un même pied, qu'ils existent légalement ou non.

Le syndicat compte, disons, cent cinquante membres. Une cinquantaine d'employés, conséquemment, sont des non-syndiqués. En vertu de quel principe de morale peut-on blâmer les cent cinquante syndiqués, s'ils décident de quitter leur emploi et d'abandonner l'employeur avec les non-syndiqués, si, en ce faisant, ils violent ni la loi, ni un contrat ? Et si cette attitude embarrasse suffisamment l'employeur pour qu'il accepte, après entente avec le syndicat, de n'employer que des syndiqués, en quoi les non-syndiqués ont-ils à se plaindre ? S'ils tiennent à rester non-syndiqués, ils doivent se résigner à ne travailler que là où les non-syndiqués peuvent être embauchés.

Dans sa brochure sur «L'Association professionnelle », publiée en 1927, M. l'abbé Maxime Fortin, ancien aumônier de la C.T.C.C., écrivait les lignes suivantes, dont l'actualité n'a pas vieilli: «Le travailleur isolé est à la merci de celui qui l'emploie. Et si tant de gens réclament qu'on ait pour lui tous les égards, ce n'est pas précisément pour son bien, mais pour le leur. »

Dans le «Catéchisme syndical », préparé par M. le chanoine Léopold Gauthier. aumônier des Syndicats catholiques de StJean, P. Q., conjointement avec M. l'abbé Georges Côté, aumô- 
nier général de la C.T.C.C., ce dernier, au chapitre de l'atelier ferme, précise comme suit la même pensée:

\& Un objectera peut-etre que les unionistes, en agissant ainsi, entravent indirectement la liberté d'embauchage de l'employeur et la liberté de travail des non-unionistes. Il est toujours permis de poser un acte bon ou indifférent de sa nature duquel s'ensuivra deux effets: l'un bon, l'autre mauvais, pourvu que l'on puisse alléguer des raisons d'une gravité proportionnée aux effets que la volonté ne peut empêcher. Les bons effets sont d'ordre professionnel et social. Les mauvais effets sont d ordre particulier ».

Mgr L.-A. Paquet, dont l'autorité, dans les domaines de la théologie, de la philosophie et de la sociologie, n’a jamais été contestée, ni de son vivant, ni depuis sa mort, écrivait de son côté: «Nous ne voulons point condamner les ouvriers syndiqués qui, sans rupture de contrat d'aucune sorte, sans danger pour lordre public ni pour le bien général de leurs familles, dans l'intérêt de l'union dont ils font partie et qu'ils jugent traitée de façon inéquitable, quittent ensemble, délibérément et pacifiquement, l'atelier où ils travaillent. En vertu de la liberté du travail dont jouissent et les individus et les unions, ils ont droit de poser au patron leurs justes conditions, de même que le patron, en acceptant le travail offert, peut poser les siennes 》. (1)

Une association patronale s'est prononcée ouvertement en faveur de l'atelier ferıné, à Washington, devant un comité parlementaire. récemment. On peut lire, en eftet, dans Newsweek, 10 février 1947, (page 67), ce qui suit: «A solid front of employers favoring legislation to outlaw the closed shop was broken last week by Victor S. Riesenfeld, chairman since 1939 of the U. S. Clothing Manufacturers Association's bargaining committee. Closed-shop contracts between clothing manufacturers and the CIO Amalgamated Clothing Workers, said Riesenfeld, have been a stabilising and constructive influence. 》

Dans un court article intitulé «The closed shop and legal authority », la revue anglaise «The Industrial Law Review》, Vol. 1, no. 7, Décembre 1946, cite une décision de la Chambre des Lords, dans la cause de Crofter Harris Tweed Co. Ltd. vs Weitch (1942). A. C. Voici l'extrait de la revue: «Here a trade union in order to prevent the employment of non-unionists being employed in the northwest of Scotland, placed an embargo on the port of Stornaway for Harris Tweed woven from mainland yarn, as opposed to the island yarn made by members of the union concerned. It was decided that this embargo was lawful, Lord Wright said: «The individual's right to freedom in conducting a trade is not absolute or unconditional; it is only a particular aspect of the citizen's right to personal freedom, and like other aspects of that right, is qualified by various legal limitations, either by Statute or by Common Law. Such limitations are inevitable in organized societies, where the rights of individuals may clash. »

Ce qui précède traite de l'atelier fermé en faveur d'un syndicat qui est en face de non-syndiqués. Mais si le Syndicat est en face, non plus de non-syndiqués, mais d'un autre ou de plusieurs autres syndicats ? Il importe de clarifier cette situation. L'atelier fermé vise à empêcher le sabotage des métiers et des salaires par les non-syndiqués, et non à supprimer le pluralisme syndical et à créer le syndicat unique et obligatoire. Il ne peut y avoir qu'un syndicat légitime et capable de défendre les intérêts professionnels des travailleurs. Dans «Catéchisme syndical », M. l'abbé Georges Côté, propose, avec raison, la solution suivante: \&Il y a lieu, dans ce cas, pour ces unions de s'entendre par le moyen du cartel. L'atelier fermé a pour but premier d'empècher les non-unionistes (scabs) de nuire à l'intérêt du métier, non d'éliminer les groupements syndicaux. Conséquemment il serait abusif de la part d'un syndicat que de tenter d'imposer l'atelier fermé contre une union organisée dans la même industrie ou le même métier. Si, d'un autre côté, cette dernière union compromet l'intérêt professionnel dans ses agissements, il est permis de la traiter comme des non-unionistes et d'user de la cessation concertée du travail pour empêcher ce groupement syndical de nuire à l'intérêt du métier. »

La sécurité syndicale, en résumé, est un phénomène social provoqué par les syndicats de travailleurs pour assurer leur sur. vivance. Ces syndicats, du moment qu'ils jouissent de leur pleine liberté, ont droit de choisir parmi les moyens qui leur paraissent les meilleurs pour atteindre leur fin. Et du moment que les moyens choisis sont bons et inattaquables devant la morale sociale, il n'appartient à personne d'intervenir pour leur imposer de choisir parmi les seuls moyens paisibles qui ne dérangent pas trop le patronat, les gouvernements et les non-syndiqués.

Les syndicats de travailleurs doivent être puissants. Leur rôle ne se borne pas seulement aux négociations collectives. Dans

(1) Semaine Sociale du Canada, deuxième session, Québec 1921, p. 46. une société bien organisée, on doit les inviter à participer à l'étude et à la solution de tous les problèmes économiques et sociaux.

En terminant, une dernière citation. Dans son rapport à la Conférence Internationale du Travail de New York (1941), le Directeur du Bureau International du Travail ne craignait pas d'affirmer:

«On sait que les problèmes sociaux et économiques ne peuvent être étudiés et traités dan; l'isolement. Si les objectifs sociaux doivent constituer l'élément fondamental de la politique générale, la participation des organisations d'employeurs et de travailleurs à tous les stades de l'établissement et de l'application de la politique économique et sociale n'est pas seulement désirable; elle est indispensable. »

Gérard PICARD

\section{LE MOUVEMENT OUVRIER CANADIEN}

«Ce volume, d'une vibrante actualité, propose à la connaissance et à la méditation du lecteur le problème du travail organisé et de son action dans les cadres de notre économie et mems audelà ». C'est en ces termes que M. Edouard Montpetit présente louvrage de M. Jean-Pierre Després: Le mouvement ouvrier canadien. (1)

Sauf pour les mitiés, il est très difficile de se débrouiller à travers les différents groupements des travailleurs au Canada. Le volume du jeune professeur de Laval, docteur en sciences sociales et itujourd'hui membre du personnel du Bureau international du Travail à Genève, vient combler un vide dans la littérature canadienne des relations du travail. On y trouve brièvement expliquée la structure du syndicalisme tant sur le plan professionnel que géographique. L'auteur brosse une esquisse historique du mouvement syndical depuis ses premiers développements jusqu'à nos jours; il décrit les principes et les tendances doctrinales de chacun des groupements importants qui se partagent l'adhésion des ouvriers canadiens: le Congrès des Métiers et du Travail, la Confédération des Travailleurs catholiques du Canada et le Congrès canadien du Travail. Enfin, il parle des aspirations politiques de la classe ouvrière et de sa participation à l'action internationale.

En appendice, M. Després touche à différents sujets en rapport avec le syndicalisme comme le marché du travail et les unions ouvrières, la carrière des relations industrielles et publie in-extenso les programmes d'après-guerre du mouvement ouvrier canadien.

Si on met de côté l'ouvrage de M. H. A. Logan, publié en langue anglaise en 1928: The History of Trade-Union Organization in Canada, qui est aujourd'hui démodé à cause de l'essor considérable qu'a pris le mouvement ouvrier surtout depuis 1939 le volume de M. Després est le seul du genre. Il faut savoir gré à l'auteur d'avoir présenté en 200 pages, d'une lecture facile et accessible à tous, les éléments nécessaires à une bonne compréhension du mouvement ouvrier canadien. Car, il ne faut pas Poublier, le syndicalisme est implanté chez nous pour y demeurer et il est un facteur important dans la vie de la nation. Ce n'est pas par ce qu'en disent les quotidiens, qui ne recherchent que le sensationnel, quand ils ne sont pas à la solde d'intérêts financiers. qu'on apprendra à le connaître sur son vrai jour. Syndiqués, employeurs et profanes trouveront un grand profit à le lire.

Gérard DION

(1) Volume de 205 pages, Fides, Montréal 1946. On peut se procurer cet ouvrage au prix de $\$ 1.50$ en s'adressant au Département des cet ouvrage au prix de $\$ 1.50$ en s'adressant au
relations industrielles, Université Laval, Québec.

\section{NOS COLLABORATEURS :}

Dion, Gérard, L. Th., L.Ph., M.Sc.Soc., professeur à la Faculté des sciences sociales de Laval et secrétaire de son Département des relations industrielles.

Drolet, Arthur, membre de l'Association professionnelle des Industriels, vice-président de F.-X. Drolet, Limitée et directeur de l'Action Sociale Limitée, Québec.

Picard, Gérard, B. A., président général de la Confédération des Travailleurs Catholiques du Canada. 